\title{
О КАПИЛЛЯРНОМ ГИСТЕРЕЗИСЕ ТЕПЛООТДАЧИ ПРИ КИПЕНИИ ЖИДКОСТИ НА ПОВЕРХНОСТИ С ПОРИСТЫМ ПОКРЫТИЕМ
}

\author{
(Представил И. Эпик)
}

В природе и технике многим процессам присуще явление гистерезиса, т. е. зависимость протекания процессов от предыстории их развития. Гистерезис теплоотдачи при кипении на гладкой поверхности подробно описан в $\left[{ }^{1}\right]$ и объяснен дополнительным расходом энергии, необходимым для активизации новых центров парообразования при увеличении плотности теплового потока. С уменьшением же тепловой нагрузки ранее активизированные центры парообразования остаются работоспособными и при меньших перепадах температур между охлаждаемой поверхностью и жидкостью.

При кипении на пористой поверхности механизм гистерезиса теплоотдачи усложняется в связи со специфнчностью процесса парообразования и особенностями движения жидкости и пара в пористом материале. В единственной известной нам работе $\left[{ }^{2}\right]$, где рассматривается гистерезис кипения на пористой поверхности, это явление объяснено возникновением медленно стабилизирующейся пленки пара в относительно толстом пористом слое. В настоящей статье рассматривается капиллярный гистерезис теплоотдачи, т. е. гистерезис, обусловленный особенностями строения пор в покрытии.

Сначала подробно проанализируем физический механизм кипения жидкости в пористом материале. Известно, что повышение температуры поверхности при кипении активизирует потенциальные центры парообразования размерами

$$
a>2 T^{\prime \prime} \sigma / r_{\varrho} \Delta T,
$$

где $a$ - радиус парового зародыша, $T^{\prime \prime}$ - температура насыщения, $\sigma$ - коэффициент поверхностного натяжения, $r-$ скрытая теплота парообразования, $\mathrm{e}$ - плотность пара, $\Delta T$ - перегрев жидкости относительно температуры насыщения.

При испарении жидкости эти зародыши пара увеличиваются, вытесняя жидкость из более крупных пор, в которых капиллярное давление сравнительно мало. Чтобы пар мог выйти из устья в большой объем жидкости, его давление должно превысить максимальное капиллярное давление жидкости для данной поры, определяемое радиусом ее минимального сечения $a_{\mathrm{M}}$ (сечения м на рис. 1). При полном смачивании это условие можем записать в виде $\Delta p>2 \sigma / a_{\mathrm{M}}$, где $\Delta p-$ разность давлений пара и жидкости. Преодолев минимальное сечение, пар полностью выталкивает жидкость из поры, пора открывается, и дальнейшее испарение жидкости происходит через остающийся на стенках пор микрослой жидкости. 


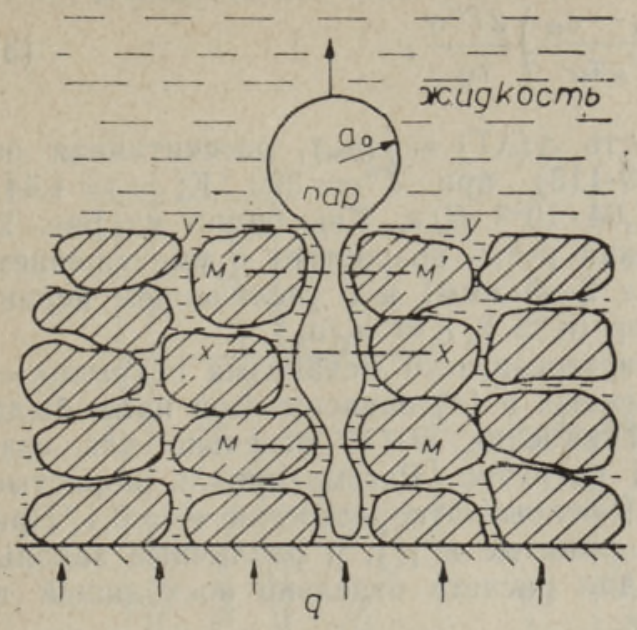

Рис. 1. Схема парообразования в пористом матернале.

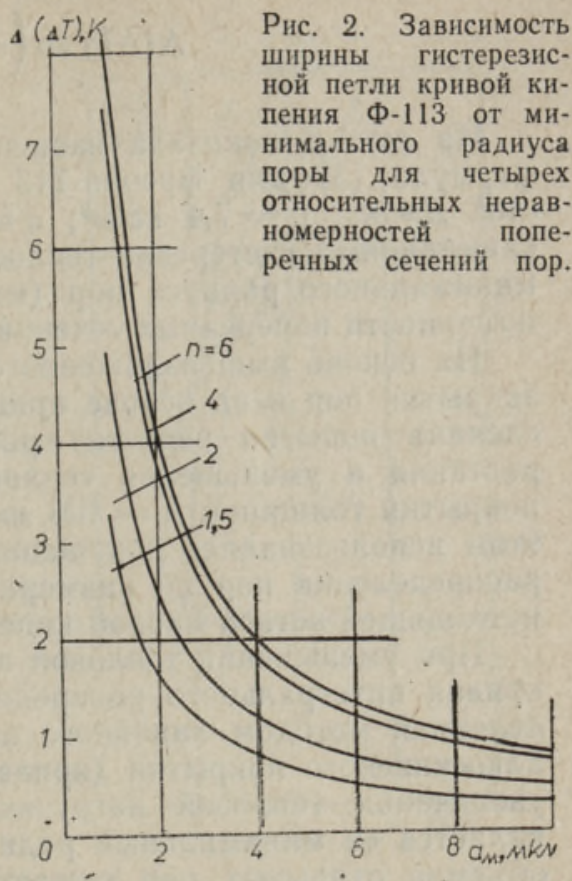

Пар выходит из поры в виде цепочки отрывающихся пузырьков. При этом давление в системе пора-пузырек $\Delta p=2 \sigma / a_{0}$, где $a_{0}$ радиус пузырька в момент отрыва. Так как в общем случае $a_{0}>a_{\mathrm{y}}$ $\left(a_{\text {y }}\right.$ - радиус устья поры), то в момент отрыва пузырька давление в заполненной паром поре меньше равновесного для выходного сечения $\Delta p=2 \sigma / a_{\mathrm{y}}$. Поэтому после отрыва пузырька жидкость под действием капиллярных сил будет проникать в. пору до момента уравновешивания этих сил давлением пара внутри поры. Используя обычное уравнение состояния для изотермического газа ( $p V=$ const) и предполагая, что скорость проникновения жидкости в пору гораздо больше скорости парообразования, можем определить глубину проникновения жидкости в единичную пору условием

$$
\Delta V=V_{n}\left[1-\left(a_{x} / a_{0}\right)\right],
$$

где $\Delta V$ - объем поры, заполненный жидкостью (между сечениями $y$ и $x$ на рис. 1$) ; V_{n}$ - полный объем поры; $a_{x}-$ радиус поры в сечении $x$.

Чтобы пар мог снова выйти из поры, его давление должно превысить $\Delta p=2 \sigma / a_{\text {м }}^{\prime}$, где $a_{\text {м }}^{\prime}$ - минимальное сечение поры на заполненном жидкостью участке. Рассмотренные выше процессы выталкивания жидкости из поры, отрыва пузырька и проникновения жидкости в пору будут постоянно повторяться. Поскольку обычно $a_{\mathrm{M}}^{\prime}>a_{\mathrm{M}}$, следовательно, для первоначального открытия поры требуется более высокое давление пара и, соответственно, более высокая температура поверхности нагрева, чем для уже открытых пор. Таким образом, причиной капиллярного. гистерезиса теплоотдачи при кипении жидкости на пористой поверхности следует считать непостоянство живого сечения пор.

Количественно ширина гистерезисной петли кривой кипения $q=f(\Delta T)$ при постоянной тепловой нагрузке $q$ для некоторой поры определяется уравнением

6 ENSV TA Toimetised. F * M 41981 


$$
\Delta(\Delta T)=\left(\frac{a_{\mathrm{M}}^{\prime}-a_{\mathrm{M}}}{a_{\mathrm{M}}^{\prime} a_{\mathrm{M}}}\right) \frac{2 T^{\prime \prime} \sigma}{r_{\mathrm{Q}}} .
$$

На рис. 2 показана зависимость $\Delta(\Delta T)=f\left(a_{\mathrm{M}}\right)$, рассчитанная по формуле (3) для фреона-113 (Ф-113) при $T^{\prime \prime}=320 \mathrm{~K} ; \quad r=1,44$. $\cdot 10^{5}$ Дж/к2; $\varrho=7,4 \kappa 2 / м^{3} ; \sigma=1,64 \cdot 10^{-2} H / \mu$. Как видно из рис. 2, капиллярный гистерезис теплоотдачи резко возрастает с уменьшением минимального радиуса пор (менее 2-3 мкм) и с увеличением неравномерности поперечных сечений пор $\left(n>2, n=a_{\mathrm{y}} / a_{\mathrm{M}}\right)$.

На основе вышеизложенного гистерезисного механизма открытия закрытия пор и на основе приведенных в $\left[{ }^{3}\right]$ зависимостей нами была сделана попытка расчета кривой кипения Ф-113 отдельно для возрастания и уменьшения тепловой нагрузки. Рассматривали пористые покрытия толщиной $L=100$ мкм и открытой пористостью $\varepsilon=0,4$. При этом использовались допущения, принятые в $\left[{ }^{3}\right]$, и различные законы распределения пор по размерам для расчета отдельно восходящей и нисходящей ветвей кривой кипения.

При уменьшении тепловой нагрузки в основу расчета была принята кривая интегрального распределения объемов пор по размерам, определенная методом линейного анализа микрошлифа образца пористого алюминиевого покрытия (кривая 4 на рис. 3). В связи с тем, что при увеличении тепловой нагрузки определяющим для открытия поры является ее минимальный радиус, кривая распределения эффективных объемов открытых пор сужается ближе к оси. Поскольку непосредственное определение этого распределения связано со значительными трудностями, оно задавалось нами произвольно (кривая 3 на рис. 3) с учетом кривой 4 .

Кипение на поверхности, покрытой пористым материалом, начинается с открытия наиболее крупных пор в точке $A$ кривой 3 (рис. 3 ). С возрастанием плотности теплового потока процесс открытия пор идет по этой кривой 3. При этом проницаемость пористого слоя для пара улучшается, а для жидкости ухудшается. Однако ухудшается до некоторого предела (к примеру, до точки $B$ ), определяемого гидродинамическим условием

$$
\frac{2 \sigma}{a_{\mathrm{M}}}=\frac{\mu_{\nVdash} \bar{w}_{\%} L}{k_{\text {ж }}}+\frac{\mu_{\Pi} \bar{w}_{\Pi} L}{k_{\mathrm{I}}} \frac{p_{1}}{\bar{p}},
$$

где $\mu-$ коэффициент динамической вязкости; $\bar{w}-$ средняя скорость движения жидкости (ж) и пара (п) в пористом слое; $k-$ проницаемость покрытия; $p_{1}$ - давление в большом объеме жидкости; $\bar{p}-$ среднее давление пара в пористом материале.

Из-за слишком малой проницаемости пористого слоя для жидкости дальнейшее возрастание тепловой нагрузки приводит к осушению некоторого участка покрытия у охлаждаемой поверхности. Следовательно, уравнение (4) определяет начало внутрислойного кризиса и вместе с тем изменение наклона кривой кипения вследствие возникновения добавочного термического сопротивления сухого пористого участка на пути теплового потока. По достижении критической плотности теплового потока покрытие осушается полностью и процесс парообразования переходит в точку $E$ (рис. 3 ). Отметим, что критическая тепловая нагрузка определяется не внутрислойным осушением покрытия, а гидродинамическими условиями в большом объеме жидкости и температурой пористой поверхности.

Расчетные кривые кипения при повышении и понижении тепловой 


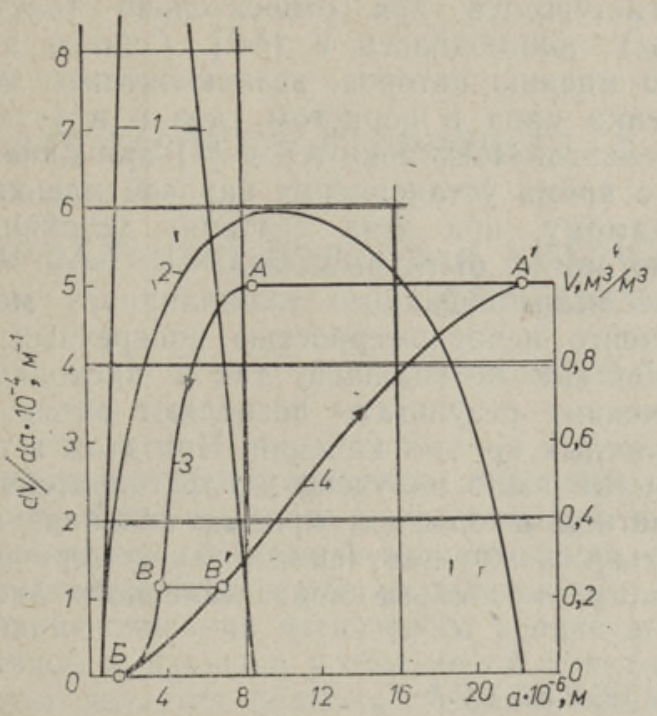

Рис. 3. Дифференциальные $(1,2)$ и интегральные $(3,4)$ кривые распределения относительного объема пор. Возрастанию тепловой нагрузки соответствуют кривые 1 и 3 , уменьшению -2 и 4 .

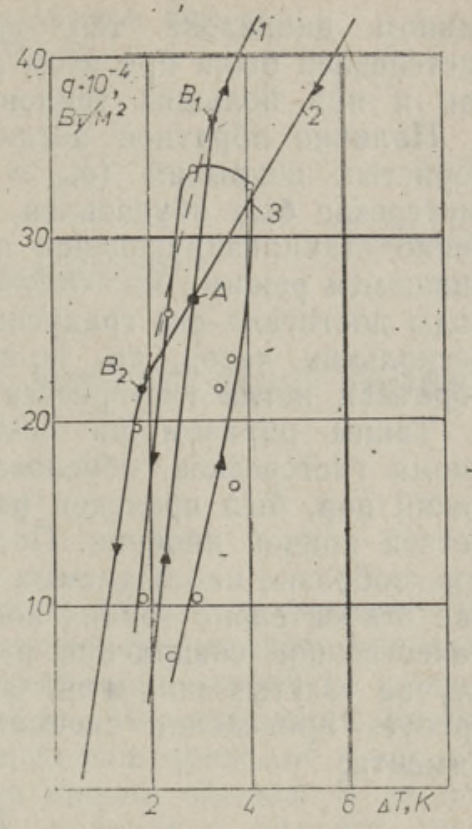

Рис. 4. Расчетные ( $I$ и 2 ) и опытная (3) кривые кипения фреона-113 на поверхности с пористым алюминиевым покрытием.

нагрузки (рнс. 4, кривые 1 и 2) имеют точки изгиба $B_{1}$ и $B_{2}$, соответствующие началу внутрислойного кризиса. При этом точка изгиба при понижении тепловой нагрузки $B_{2}$ располагается ниже точки изгиба, соответствующей возрастанию теплового потока $B_{1}$. Под влиянием капиллярного гистерезиса впитывания жидкости в пористый слой кривые 1 и 2 перекрещиваются в некоторой точке $A$. Переход от кривой 1 к кривой 2 и обратно при изменении направления процесса происходит таким образом, что проницаемость покрытия (или толщина сухого пористого слоя при $q>q_{B}$ ) не изменяется. К примеру, на рис. 4 показан пунктиром переход из точки $B_{1}$ в точку $B_{2}$, который происходит при уменьшении плотности теплового потока.

Полученное расположение восходящей и нисходящей ветвей расчетных кривых кипения позволяет объяснить большое разнообразие наблюдаемых опытных кривых. При этом определяющим становится соотношение критической плотности теплового потока для покрытия $q_{\text {кр }}$ и ординаты точки изгиба восходящей ветви $q_{B_{1}}$. Если для относительно тонких пористых покрытий, для которых $q_{\text {кр }}<q_{B_{1}}$, обязательно наличие только нижней петли гистерезиса, то для толстых покрытий $\left(q_{\text {кр }}>q_{B_{1}}\right)$ и больших тепловых нагрузок может иметь место и обратная петля гистерезиса.

Первый случай $\left(q_{\mathrm{kp}}<q_{B_{1}}\right)$ наблюдался нами опытным путем при кипении Ф-113 на горизонтальной алюминиевой поверхности с пористым покрытием, полученным методом плазменного напыления алюминиевого порошка (см. кривую 3 на рис. 4). Описание опытной установки и методики эксперимента приведено в $\left[{ }^{4}\right]$. Толщина покрытия составляла 110 мкм, открытая пористость - 0,29 и средний размер пор - 4,2 мкм. Гистерезис теплоотдачи наблюдался во всем исследо- 
ванном диапазоне тепловых нагрузок $(7-33) \cdot 10^{4} B T / \mu^{2}$. Петля гистерезиса была при этом почти одинаковой ширины как при малых, так и при больших тепловых нагрузках.

Наличие обратной петли гистерезиса для относительно толстых пористых покрытий $\left(q_{\text {кр }}>q_{B_{1}}\right)$ наблюдалось в $\left[{ }^{2,5}\right]$. Однако этот гистерезис был обусловлен, по мнению авторов, возникновением медленно стабилизирующейся пленки пара в пористом слое и в установившемся режиме мог отсутствовать. Поскольку $\Delta T$ в $\left[{ }^{2}\right]$ при кипении воды достигала ста градусов, а время установления паровой пленки нескольких часов, то, по-видимому, при этих условиях устойчивая обратная петля гистерезиса не могла быть выявлена.

Таким образом, на основе вышеописанного капиллярного механизма гистерезиса, обусловленного неравномерностью поперечных сечений пор, был проведен расчет как восходящей, так и нисходящей ветвей кривой кипения. Полученные результаты позволяют объяснить многообразие наблюдаемых опытных кривых кипения. При этом в случае относительно тонких покрытий было получено удовлетворительное качественное совпадение расчетной и опытной кривых кипения, а в случае толстых покрытий подтверждение полученных закономерностей требует проведения специального, тщательно подготовленного эксперимента.

\section{Л И ТЕ Р А Т У Р А}

1. Григорьев В. А., Павлов Ю. М., Аметистов Е. В., Кипение криогенных жидкостей, М., «Энергия», 1977.

2. Андрианов А. Б., Малышенко С. П., Сиренко Е. И., Стырикович М. А., Докл. АН СССР, 256, № 3, 591-595 (1981).

3. Техвер Я., Т уник А., Изв. АН ЭССР, Фнз. Матем., 28, № 1, 68-72 (1979).

4. Т ех ве р Я., Т ун ик А., Изв. АН ЭССР, Физ. Матем., 26, № 2, 194-198 (1977).

5. Ковалев С. А., Леньков В. А., Теплоэнергетика, № 4, 8-10 (1981).

Институт термофизики и электрофизики Академии наук Эстонской ССР

Поступила в редакцию $27 / \mathrm{V} 1981$

\section{J. TEHVER, H. SUI, R. LÄANE}

\section{SOOJUSOLEKANDE KAPILLAARNE HUSTEREES VEDELIKU KEEMISEL POORSE KATTEGA PINNAL}

On vaadeldud pooride ebaühtlasest elavristlōikest tingitud soojusülekande hüstercesi füüsikalist mehhanismi vedeliku keemisel poorsel kattel ning arvutatud keemiskōvera tõusu- ja langusharu. Tulemusi on võrreldud katseandmetega.

\section{J. TEHVER, H. SUI, R. LAANE}

\section{ON A CAPILLARY HYSTERESIS OF A FLUID BOILING ON THE SURFACE OF A POROUS COATING}

Physical mechanism of heat exchange hysteresis conditioned by the cross section irregularity of a pore for a boiling fluid with a porous coating has been considered.

According to the analysis, the capillary hysteresis increases with the decrease of minima dimensions of pores smaller than $3 \mu \mathrm{m}$ and with cross section irregularities of a pore for $n>2$.

On the basis of this data, the upward and downward branches of a boiling curve were calculated independently. The obtained results allow to explain the considerable diversity of the observed experimental boiling curves. Therewith, the calculated and experimental boiling curves were found to be in a good qualitative argument for relatively thin coatings. However, a special well-prepared experiment must be carried out for thick coatings because of a slowly stabilizing vapor film in a porous material. 